\title{
How bacteria hack the matrix and dodge the bullets of immunity
}

\author{
Magnus Paulsson ${ }^{1,2}$ and Kristian Riesbeck ${ }^{2}$ \\ Affiliations: ${ }^{1}$ Division for Infectious Diseases, Skåne University Hospital, Lund, Sweden. ${ }^{2}$ Clinical Microbiology, \\ Dept of Translational Medicine, Faculty of Medicine, Lund University, Malmö, Sweden.
}

Correspondence: Kristian Riesbeck, Clinical Microbiology, Dept of Translational Medicine, Faculty of Medicine, Lund University, Jan Waldenströms gata 59, SE-205 02 Malmö, Sweden. E-mail: kristian.riesbecklamed.lu.se

\section{@ERSpublications}

Bacterial pathogens adhere to human proteins in the lung to avoid being washed out. This can potentially be used as a strategy for therapeutical interventions. http://ow.ly/TW4P30kgGIB

Cite this article as: Paulsson M, Riesbeck K. How bacteria hack the matrix and dodge the bullets of immunity. Eur Respir Rev 2018; 27: 180018 [https://doi.org/10.1183/16000617.0018-2018].

ABSTRACT Haemophilus influenzae, Moraxella catarrhalis and Pseudomonas aeruginosa are common Gram-negative pathogens associated with an array of pulmonary diseases. All three species have multiple adhesins in their outer membrane, i.e. surface structures that confer the ability to bind to surrounding cells, proteins or tissues. This mini-review focuses on proteins with high affinity for the components of the extracellular matrix such as collagen, laminin, fibronectin and vitronectin. Adhesins are not structurally related and may be lipoproteins, transmembrane porins or large protruding trimeric auto-transporters. They enable bacteria to avoid being cleared together with mucus by attaching to patches of exposed extracellular matrix, or indirectly adhering to epithelial cells using matrix proteins as bridging molecules. As more adhesins are being unravelled, it is apparent that bacterial adhesion is a highly conserved mechanism, and that most adhesins target the same regions on the proteins of the extracellular matrix. The surface exposed adhesins are prime targets for new vaccines and the interactions between proteins are often possible to inhibit with interfering molecules, e.g. heparin. In conclusion, this highly interesting research field of microbiology has unravelled host-pathogen interactions with high therapeutic potential.

\section{Introduction}

40 years ago, KuUSELA [1] reported that Staphylococcus aureus binds fibronectin to the bacterial cellular surface. During the following 40 years, research has aimed to discover vaccines and antimicrobial drug targets and has revealed intricate interactions between pathogens and the extracellular matrix (ECM).

Microbes enter the airway with inhaled air or by aspiration. Some bacteria reach the lower respiratory tract where the rich amount of nutrients, humidity and constant temperature fulfil most requirements for growth. The epithelium and mucus form the first host defence barrier against invading pathogens. Ciliated bronchial epithelial cells are covered by a thin fluid layer with the tips of the cilia reaching into the viscous mucus layer, propelling mucus and any trapped bacteria towards the oropharynx with their beats, where they are swallowed or expectorated [2]. Bacterial pathogens adhere to epithelial cells or any exposed ECM in order to prevent removal. Adherence is achieved by adhesins, which are bacterial surface structures that confer the ability to bind to surrounding cells, proteins or tissues. Both pili and flagella are adhesins, although adhesins may also be transmembrane proteins or lipoproteins. Bacterial adherence is a crucial step in bacterial colonisation and a prerequisite for subsequent invasion into host cells and further dissemination in the body.

The lungs of patients with chronic obstructive pulmonary disease (COPD) are commonly infected or in some cases colonised by Gram-positive Streptococcus pneumoniae or the Gram-negative Haemophilus

Commissioned article, peer reviewed.

Received: March 022018 | Accepted after revision: May 292018

Copyright OERS 2018. ERR articles are open access and distributed under the terms of the Creative Commons Attribution Non-Commercial Licence 4.0. 
influenzae, Moraxella catarrhalis and Pseudomonas aeruginosa [3]. This mini-review focuses on the three Gram-negative bacterial species. H. influenzae and M. catarrhalis are human-specific pathogens that cause acute otitis media, sinusitis and bronchitis, and are frequent commensals in the human respiratory tract. Since the introduction of general immunisation programmes against $H$. influenzae type b (Hib), nontypeable $H$. influenzae (NTHi) has become an increasingly common pathogen [4]. In addition to infecting COPD patients during exacerbations, . aeruginosa is feared for long-term colonisation of the lungs of cystic fibrosis patients [5].

The ECM is a dynamic meshwork of proteins surrounding cells in all mammalian tissues, with a composition that changes depending on external factors or injury. The main components are proteoglycans (e.g. heparan sulfate, perlecan and agrin), soluble glycoproteins (vitronectin and fibronectin) and fibrous proteins (collagen, elastin and laminin). These proteins cement cells together and organise them into tissues with different compositions and properties [6].

ECM proteins have multiple binding sites for cellular surface receptors and other ECM proteins. For instance, heparin-binding domains (HBDs) interact with cell-surface-bound heparan sulfate [7]. This domain is typically involved in ECM-bacterial interactions, suggesting that nebulised heparin theoretically may have a therapeutic role. Integrins act as cell surface receptors that anchor cells to the ECM and also link the ECM to intracellular signalling pathways. The integrins consist of heterodimers with one $\alpha$ and one $\beta$ subunit that bind specifically to one or several protein domains. These surface molecules enable soluble glycoproteins to form links between the larger fibrils and cells, and to initiate cellular responses to changes in the surrounding environment.

Smoking, inflammation, viral infections and mechanical ventilation disrupt the respiratory epithelium and expose patches of ECM that are recognised by bacterial adhesins [8]. The ECM composition is also altered by smoking or chronic lung diseases. The laminin layer in basal laminas of smokers is thicker, and patients with COPD have increased bronchial deposition of fibronectin, laminin and collagen [9, 10]. Moreover, sarcoidosis or interstitial lung diseases are associated with elevated vitronectin and fibronectin levels in bronchoalveolar lavage fluid $[11,12]$.

TABLE 1 A few examples of bacterial adhesins in Gram-negative bacteria

\begin{tabular}{|c|c|c|c|c|c|c|}
\hline \multirow{2}{*}{$\begin{array}{l}\text { ECM components } \\
\text { interacting with }\end{array}$} & \multicolumn{2}{|r|}{ NTHi adhesins } & \multicolumn{2}{|r|}{ M. catarrhalis adhesins } & \multicolumn{2}{|r|}{$P$. aeruginosa adhesins } \\
\hline & Name & Characteristics & Name & Characteristics & Name & Characteristics \\
\hline \multirow[t]{2}{*}{ Collagen } & Hap & $\begin{array}{l}\text { Kd } 20 \mathrm{nM} \text { (ELISA), } \\
\text { auto-transporter [14] }\end{array}$ & $\begin{array}{l}\text { MID/ } \\
\text { Hag }\end{array}$ & Auto-transporter [15] & & \\
\hline & & & UspA2 & $\begin{array}{l}\text { Trimeric auto-transporter } \\
{[16,17]}\end{array}$ & & \\
\hline Fibronectin & Hap & $\begin{array}{l}\text { Kd } 15 \mathrm{nM} \text { (ELISA), } \\
\quad \text { auto-transporter [14] }\end{array}$ & UspA1 & $\begin{array}{l}\text { Trimeric auto-transporter } \\
{[16,18]}\end{array}$ & OprQ & OprD-family porin [19] \\
\hline \multirow[t]{2}{*}{ Vitronectin } & Protein $\mathrm{E}$ & $\begin{array}{l}\text { Kd } 400 \mathrm{nM} \text { (surface } \\
\text { plasmon resonance), } \\
\text { lipoprotein }[21,22]\end{array}$ & UspA2 & $\begin{array}{l}\text { Kd } 23 \mathrm{nM} \text { (surface plasmon } \\
\text { resonance), trimeric } \\
\quad \text { auto-transporter }[16,23,24]\end{array}$ & OprD & $\begin{array}{l}\text { Kd } 3.6 \mathrm{nM} \text { (biolayer } \\
\text { interferometry), } \\
\text { OprD-family porin [25] }\end{array}$ \\
\hline & Protein $\mathrm{F}$ & $\begin{array}{l}\text { Kd } 12.8 \mathrm{nM} \text { (ELISA), ABC } \\
\text { transporter [26] }\end{array}$ & & & Lpd & Moonlighting protein [27] \\
\hline \multirow{2}{*}{ Laminin } & Protein $\mathrm{E}$ & $\begin{array}{l}\text { Kd } 1.5 \mu \mathrm{M} \text { (surface } \\
\text { plasmon resonance), } \\
\text { lipoprotein }[22,30]\end{array}$ & UspA2 & $\begin{array}{l}\text { Trimeric auto-transporter } \\
\quad[16,28]\end{array}$ & & \\
\hline & $\begin{array}{l}\text { Protein F } \\
\text { P4 }\end{array}$ & $\begin{array}{l}\text { ABC transporter [31] } \\
\text { Kd } 9.3 \text { nM (ELISA), } \\
\text { lipoprotein [20] }\end{array}$ & AfeA & Protein F orthologue [29] & & \\
\hline
\end{tabular}

ECM: extracellular matrix; NTHi: nontypeable Haemophilus influenzae; M. catarrhalis: Moraxella catarrhalis; P. aeruginosa: Pseudomonas aeruginosa; Hap: Haemophilus adhesion and penetration protein; $K d$ : dissociation constant for adhesin-ECM interaction; MID: M. catarrhalis IgD-binding protein; Usp: ubiquitous surface protein; Opr: outer membrane porin; ABC: ATP-binding cassette; Lpd: dihydrolipoamide dehydrogenase. 
Three different interactions between respiratory tract bacteria and the ECM are known: 1) bacterial adhesion to the ECM, 2) degradation of the ECM by bacterial proteases, and 3) antibacterial action of fragments of ECM proteins that are formed as these proteins are cleaved [13]. This mini-review focuses on adhesion, and examples of known adhesins are listed in table 1. Most recent studies describe the nature of the interactions, phenotypes of mutated bacteria and in some cases structural data of the full or truncated proteins, which will only be briefly reported here.

\section{Bacterial interactions with collagen}

Collagens are the most abundant proteins in the lung ECM [32]. They are the principal tensile element of tissues, shaped as elongated fibrils. Several $\alpha$-chain monomers associate into a triple-helix form, which further associates to create various fibrillary or network arrangements [33]. The collagens are numbered with Roman digits according to their discovery and correspond to unique proteins composed of one, two or three gene products. Types I, II, III, IV and VI are of relevance in the respiratory tract. Type I collagen is present in all human tissues, whereas type II collagen associates with cartilage, including trachea. Type III collagen is present in connective tissues and the network-forming type IV is a major component of the
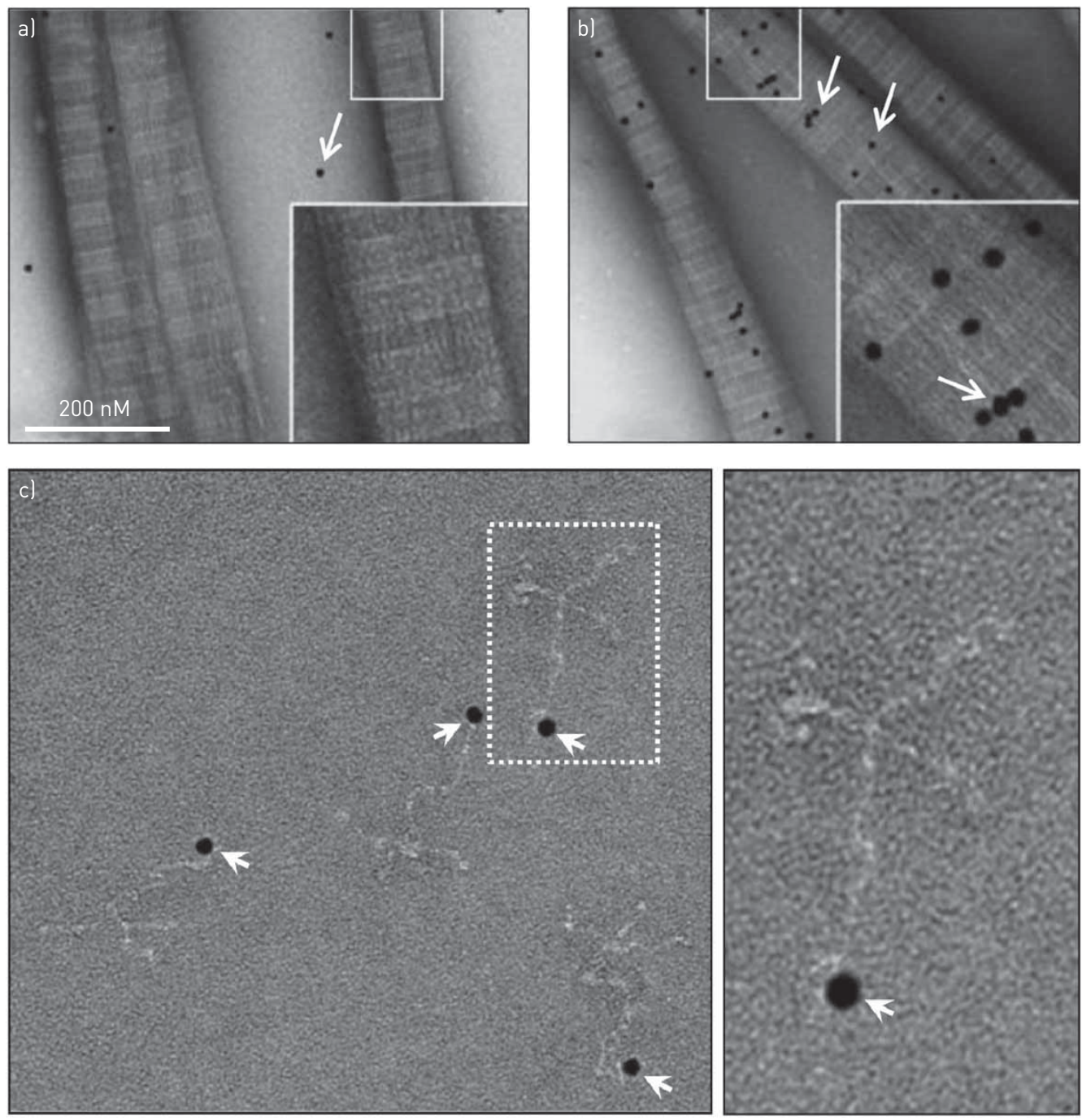

FIGURE $1 \mathrm{a}$ and b) Moraxella catarrhalis ubiquitous surface protein (Usp) A2 binds collagen with high affinity. Transmission electron microscopy (TEM) demonstrates that al gold-labelled recombinant UspA1 does not bind to collagen I fibrils, whereas b) UspA2 does. Reproduced and modified from [17] with permission. c) Bacterial adhesins target laminin globular domains at the base of the asymmetrical cross-shaped laminin. TEM image showing gold-labelled Protein F from nontypeable Haemophilus influenzae bound to laminin. Reproduced and modified from [31] with permission; these images are not included under the Creative Commons CC BY-NC 4.0 licence of the current article. In all panels, gold-labelled recombinant bacterial proteins are marked with white arrows. 
basal lamina that anchors epithelial cells to the mesenchyme. Finally, collagen type VI is abundantly present in the ECM of the respiratory tract and, like collagens I and III (in addition to laminin), is upregulated in COPD [17]. During epithelial damage, collagens are exposed in the airway mucosa and accessible to intruding bacteria [10]. However, only a few pathogens have been described to bind collagen directly. The majority use surface-bound fibronectin as a bridging molecule [34].

Clinical H. influenzae isolates are known to bind collagen I and IV [35]. NTHi binds to collagen using Haemophilus adhesion and penetration protein (Hap), an adhesin that belongs to the auto-transporters. Hap promotes adhesion to epithelial cells and causes bacterial aggregation, which facilitates colonisation of the respiratory mucosa. It also mediates bacterial invasion, leading to an intracellular bacterial reservoir that may be responsible for recurrent infections seen in COPD [36]. Hap has a $\beta$-barrel domain anchoring it in the outer membrane and a protruding passenger domain with protease activity. It binds multiple ECM components simultaneously and binds to laminin and fibronectin using different domains [14, 37].

All tested M. catarrhalis clinical isolates bind collagens I, II, III, IV and VI, but the binding capacity varies between isolates. M. catarrhalis IgD-binding protein (MID, also known as Hag) is structurally similar to Hap and has been reported to bind collagen $[15,17]$. MID also confers adherence to type II alveolar cells and is highly immunogenic. However, recent evidence suggests that the ubiquitous surface protein (Usp) $\mathrm{A} 2$ and the related UspA2H are more important for collagen binding (figure 1a and b) [17]. The UspAs are trimeric auto-transporter adhesins with a lollipop structure. A membrane anchor is embedded in the outer membrane, and the stalk, neck and head domains extend outwards $800 \AA$ from the cell, where they form a densely packed molecular coating [16]. The UspAs bind several components of the ECM and are crucial for the virulence of $M$. catarrhalis (figures 2 and 3). Mice with smoke-induced COPD are

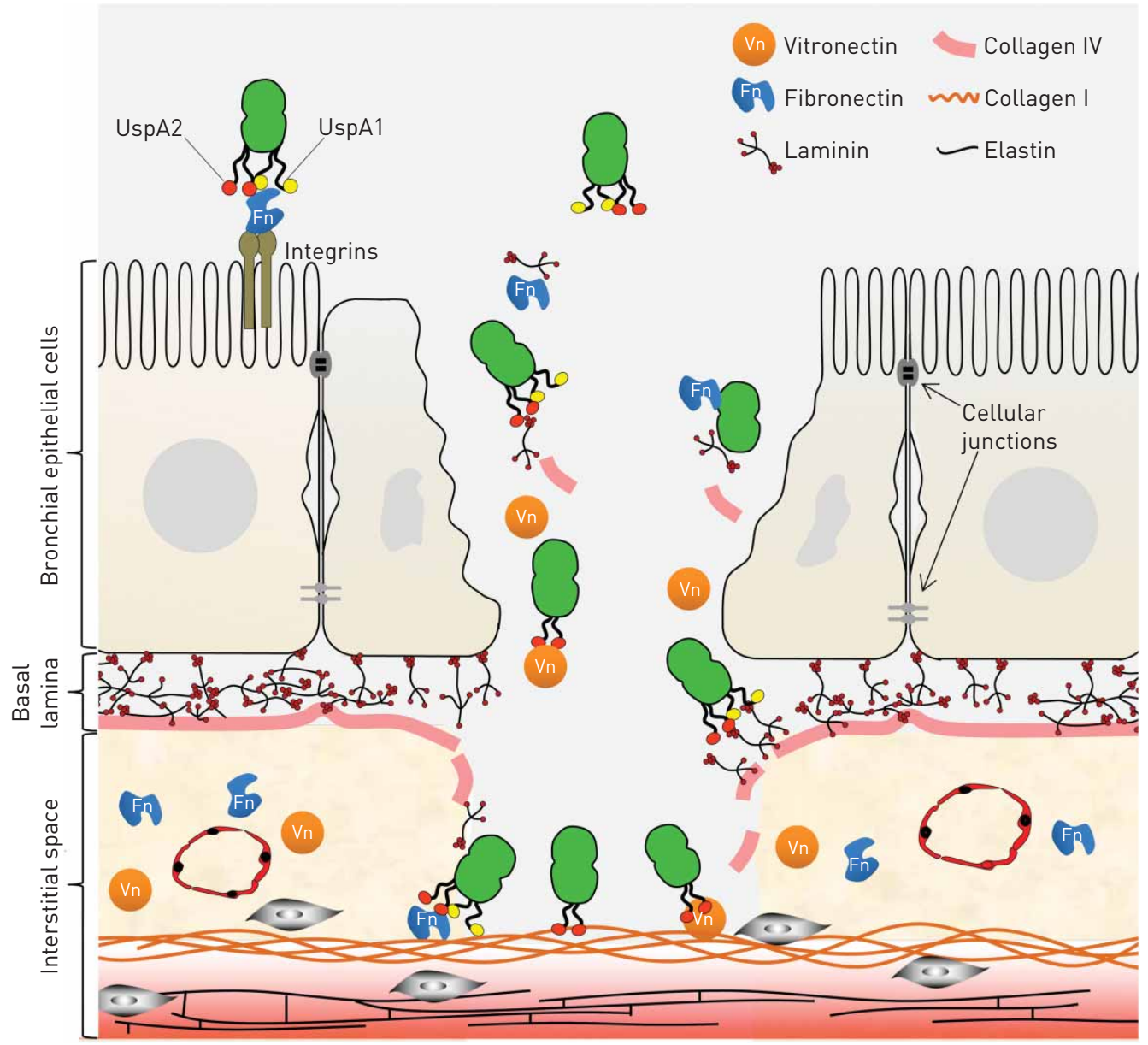

FIGURE 2 Moraxella catarrhalis adheres to the extracellular matrix in the respiratory tract. Cartoon that illustrates a disrupted bronchial epithelium and how M. catarrhalis colonises the human lung by using ubiquitous surface protein (Usp) A1 and UspA2 to adhere to laminin, collagen, fibronectin (Fn) and vitronectin (Vn). For simplicity, MID and AfeA were omitted from this cartoon. Reproduced and modified from [17] with permission. 
colonised about four times less efficiently by mutated bacteria lacking UspA2 [17]. Most patients with COPD have high titres of antibodies directed both against the adhesive domain of MID and the ECM-binding domains of UspA1/A2 [38]. However, the binding regions of $M$. catarrhalis UspAs are highly diverse; thus, the bacterium evades recognition by IgG and complement-mediated attacks [39].

$P$. aeruginosa adheres to collagen I, tracheal collagen II and collagen IV in basal lamina, but surface receptors are yet to be described. $P$. aeruginosa collagen binding is inhibited by heparin, suggesting binding to a conserved heparin-binding domain of the collagen molecule [40]. During long-term $P$. aeruginosa colonisation, adherence to sessile ECM or cells may be of less importance than "hiding" in the self-produced biofilm and binding to mucin, for which it has high affinity [41]. Exposing adhesins may in this setting be counterproductive, as maintaining adhesion needs to be balanced against detection by the immune system.

\section{Fibronectin as a bacterial target}

Fibronectin is a soluble multi-adhesive matrix protein. Its primary role is to attach cells to other components of the ECM, especially collagen. Most bacterial pathogens have evolved strategies to utilise fibronectin for adhesion to cells or to collagen by using fibronectin as a bridging molecule. Fibronectin attaches to the cell surface as fibrils that are continuously replaced or endocytosed. Bacteria with fibronectin on the surface exploit the same mechanism to become internalised by human cells [42]. However, macrophages phagocytose fibronectin-covered bacteria and internalisation poses a threat to the bacteria, although, for example, NTHi is able to replicate and attract nutrients intracellularly [43].

NTHi binds fibronectin using Hap and lipoprotein P4, although the initial reports indicated that a pilus was responsible for the binding $[14,20]$. P4 is a lipoprotein that is involved in nicotinamide adenine dinucleotide uptake and haemin utilisation, both essential nutrients for this species. P4 binds ECM components, including laminin and vitronectin, using a central $\alpha$-helix. In addition, P4-dependent NTHi adhesion is observed to type II alveolar cells and bronchial epithelium. P4 is necessary for long-term survival in mouse lung and mutant strains devoid of $\mathrm{P} 4$ are attenuated in the otitis media Junbo mouse model $[20,44]$.

M. catarrhalis uses both UspA1 and UspA2 for fibronectin binding [18]. Intriguingly, during "cold shock" $\left(26^{\circ} \mathrm{C}\right)$, the UspAs are upregulated. This facilitates bacterial colonisation by enhancing the adhesive properties of $M$. catarrhalis at physiologically relevant temperatures in the upper respiratory tract [45].

The genome of $P$. aeruginosa is almost four times larger than that of NTHi or $M$. catarrhalis, causing a redundancy of adhesins. Already by 1993 it was discovered that $P$. aeruginosa uses fibronectin for adherence to collagen. In 2002, at least six fibronectin-binding proteins were suggested. Eventually, OprQ (outer membrane porin Q), a porin belonging to the OprD superfamily, was identified as a fibronectin-binding adhesin [19]. The OprDs are $\beta$-barrel-shaped transmembrane proteins that transport
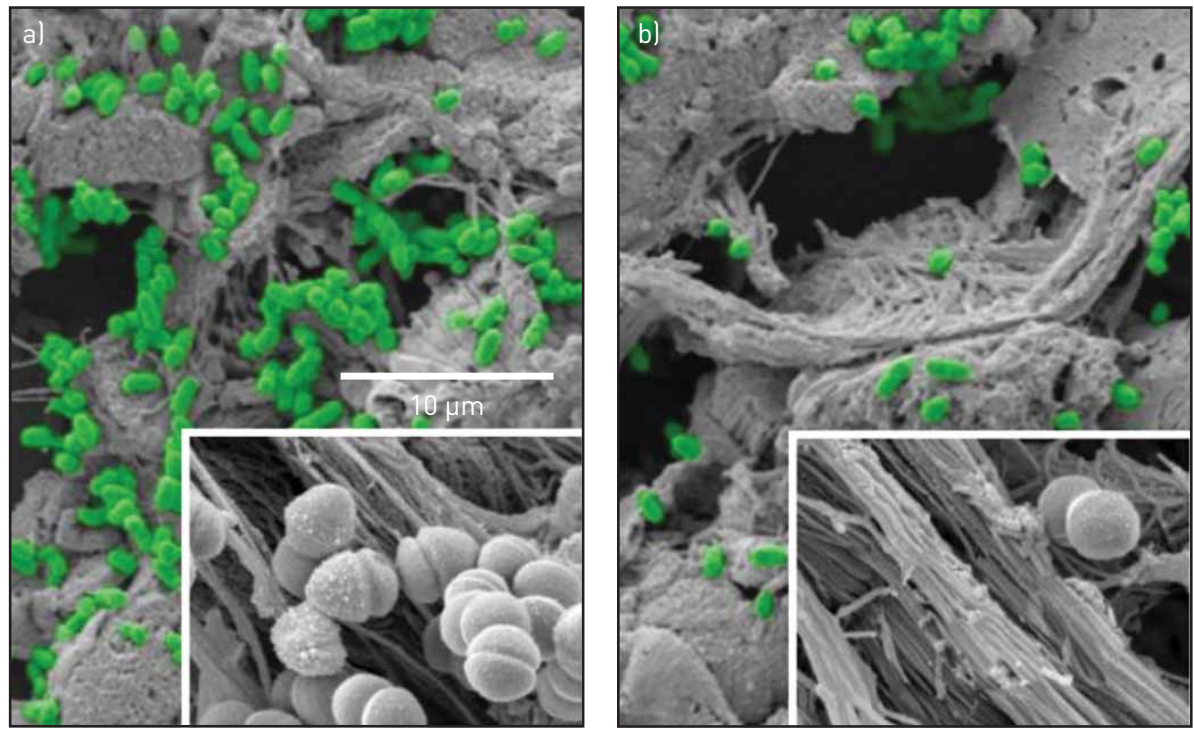

FIGURE 3 Scanning electron micrographs showing that a) Moraxella catarrhalis bacteria (in green pseudocolourl adhere to human tracheal specimens, whereas b) UspA2-deficient mutants do not. Reproduced and modified from [17] with permission. 
nutrients into the cell. Imipenem enters the periplasm through $\mathrm{OprD}$, which is frequently mutated in carbapenem-resistant strains [25].

\section{Vitronectin is used by respiratory pathogens in various ways}

The glycoprotein vitronectin is mainly produced in the liver, although bacterial stimulation triggers local production in the respiratory tract by alveolar epithelial cells [46]. More than a "cell glue", vitronectin plays homeostatic roles and regulates the terminal pathway of the complement cascade, where it inhibits formation of the cytolytic membrane attack complex (MAC). Most Gram-negative bacteria are sensitive to lysis by MAC and are killed by it unless complement regulators are recruited at the bacterial surface, i.e. they are serum resistant. Escaping complement by recruiting complement regulators is thus a key characteristic for respiratory pathogens. The majority of tested pathogenic bacteria recruit vitronectin to the cellular surface using the same C-terminal HBD3, which is inhibited by low concentrations of heparin [47].

Similarly to fibronectin, vitronectin is used as a bridging molecule between bacteria and human cells. NTHi adheres to bronchial epithelial cells via vitronectin using Protein E [48]. This adhesin is a conserved lipoprotein in the Pasteurellaceae family and other unrelated species (e.g. Enterobacter cloacae and Listeria monocytogenes) [22]. Although Protein E is a high-affinity adhesin, mutants devoid of Protein E have residual vitronectin-binding capacity. Protein $\mathrm{F}$ and $\mathrm{P} 4$ have since been identified as additional vitronectin receptors in NTHi [20, 21, 26].

The main vitronectin receptor in $M$. catarrhalis is the lollipop-shaped UspA2 [16, 23]. Mutated bacteria lacking UspA2 are highly sensitive to killing by human serum [23]. The interaction between UspA2 and vitronectin is independent of ionic strength and heparin inhibition [24]. A recent study comparing vitronectin-binding capacity of several bacterial species reported that $M$. catarrhalis and group A streptococci have higher vitronectin-binding capacity than other species tested [45].

$P$. aeruginosa also utilises vitronectin for adhesion to mammalian cells and to evade complement-mediated attack. Two vitronectin receptors for $P$. aeruginosa are known, OprD and dihydrolipoamide dehydrogenase (Lpd) [25, 27]. Lpd is involved in pyruvate metabolism in the cytoplasm, but is also exposed on the bacterial surface where it binds multiple complement regulators and components of the ECM.

\section{Laminin is an important molecule for bacterial attachment}

Laminins are large ECM proteins $(\sim 800 \mathrm{kDa})$ and major components of the basal lamina. They are composed of three polypeptide chains $(\alpha, \beta$ and $\gamma)$, which together form an asymmetrical cross containing five globular domains and a coiled-coil region. The globular domains interact with a range of integrins, heparin sulfate proteoglycans and other receptors, contributing to cell-ECM attachment but also to cell shape and differentiation, maintenance of tissue phenotypes and to the stability and integrity of the basal lamina. There are several laminin isoforms. Embryonic laminin-111 $(\alpha 1, \beta 1$ and $\gamma 1)$ is the isoform used in all cited papers. Based on tissue-specific localisation, laminin $\alpha 5$ is, however, more relevant to include in future studies since this isoform appears in the lung [32].

To date, NTHi has four known laminin-binding adhesins: Hap, Protein E, Protein F and P4 [14, 20, 30, 31]. All of these are multifunctional ECM-binding proteins but share no sequence similarity with each other, although both Protein E and Protein F bind to the laminin globular domains 4-5 (figure 1c) [31]. These globular domains contain the binding sites for heparin; hence, the interaction with these bacterial adhesins competes with heparin.

M. catarrhalis binds laminin using UspA1 and UspA2. While the "head" of the UspA molecules interacts with laminin, the stalks interact with fibronectin, both contributing to adhesion [28]. In addition, M. catarrhalis, $P$. aeruginosa and several other species have Protein F orthologues (e.g. AfeA in M. catarrhalis and Paf in P. aeruginosa) that interact with the globular domains [29].

\section{Concluding remarks}

Respiratory bacteria interact with the ECM through a multitude of adhesins with overlapping functions. Adherence to the ECM is a conserved mechanism among pathogenic bacteria and is most likely to be important during colonisation of the respiratory tract.

The prospect of developing adhesin-based vaccines has motivated the research field. There are examples from other bacterial species where surface proteins have been successfully used in vaccinology, e.g. factor H-binding protein in Neisseria meningitidis serogroup B vaccine. NTHi has received more attention after the introduction of a Hib vaccine, and several of the adhesins listed in table 1 have been or are currently being evaluated for their immunogenic and protective properties. Considering the urgency for a vaccine against $P$. aeruginosa, it is surprising that less is known about adhesins in that species than in $M$. 
catarrhalis. There have, however, been clinical trials on vaccines against $P$. aeruginosa, but they have hitherto been unsuccessful.

As most bacterial adhesins target HBDs in the ECM proteins, the use of nebulised heparin for patients with high risk of pulmonary infections is theoretically tempting. In a recent meta-analysis, three out of four studies reported increased bacterial clearance after nebulised heparin was administered [49]. Unfortunately, this did not protect against ventilator-associated pneumonia or hasten recovery from pneumonia in patients receiving mechanical ventilation [50].

In summary, much effort has been made in exploring the interactions between the ECM and respiratory bacteria and several possible vaccine targets and targetable host-pathogen interactions have been suggested. The next few years will tell if the therapeutic potential can be translated into clinically useful vaccines or antimicrobial drugs.

Conflict of interest: None declared.

Support statement: Funding support was received from Anna and Edwin Berger, the Swedish Medical Research Council (grant number K2015-57X-03163-43-4), the Cancer Foundation at the University Hospital in Malmö, Skåne County Council's research and development foundation, the Heart Lung Foundation (Stockholm, Sweden; grant number 20150697) and the Royal Physiographical Society (Lund, Sweden). Funding information for this article has been deposited with the Crossref Funder Registry.

\section{References}

$1 \quad$ Kuusela P. Fibronectin binds to Staphylococcus aureus. Nature 1978; 276: 718-720.

2 Widdicombe JH, Bastacky SJ, Wu DX, et al. Regulation of depth and composition of airway surface liquid. Eur Respir J 1997; 10: 2892-2897.

3 Wang Z, Bafadhel M, Haldar K, et al. Lung microbiome dynamics in COPD exacerbations. Eur Respir J 2016; 47: 1082-1092.

4 Clementi CF, Murphy TF. Non-typeable Haemophilus influenzae invasion and persistence in the human respiratory tract. Front Cell Infect Microbiol 2011; 1: 1.

5 Millares L, Ferrari R, Gallego M, et al. Bronchial microbiome of severe COPD patients colonised by Pseudomonas aeruginosa. Eur J Clin Microbiol Infect Dis 2014; 33: 1101-1111.

6 White ES. Lung extracellular matrix and fibroblast function. Ann Am Thorac Soc 2015; 12: Suppl. 1, S30-S33.

7 Singh B, Fleury C, Jalalvand F, et al. Human pathogens utilize host extracellular matrix proteins laminin and collagen for adhesion and invasion of the host. FEMS Microbiol Rev 2012; 36: 1122-1180.

8 de Bentzmann S, Plotkowski C, Puchelle E. Receptors in the Pseudomonas aeruginosa adherence to injured and repairing airway epithelium. Am J Respir Crit Care Med 1996; 154: S155-S162.

9 Amin K, Ekberg-Jansson A, Löfdahl CG, et al. Relationship between inflammatory cells and structural changes in the lungs of asymptomatic and never smokers: a biopsy study. Thorax 2003; 58: 135-142.

10 Kranenburg AR, Willems-Widyastuti A, Moori WJ, et al. Enhanced bronchial expression of extracellular matrix proteins in chronic obstructive pulmonary disease. Am J Clin Pathol 2006; 126: 725-735.

11 Eklund AG, Sigurdardottir O, Ohrn M. Vitronectin and its relationship to other extracellular matrix components in bronchoalveolar lavage fluid in sarcoidosis. Am Rev Respir Dis 1992; 145: 646-650.

12 Pohl WR, Conlan MG, Thompson AB, et al. Vitronectin in bronchoalveolar lavage fluid is increased in patients with interstitial lung disease. Am Rev Respir Dis 1991; 143: 1369-1375.

13 Andersson E, Rydengård V, Sonesson A, et al. Antimicrobial activities of heparin-binding peptides. Eur J Biochem 2004; 271: 1219-1226.

14 Fink DL, Buscher AZ, Green B, et al. The Haemophilus influenzae Hap autotransporter mediates microcolony formation and adherence to epithelial cells and extracellular matrix via binding regions in the C-terminal end of the passenger domain. Cell Microbiol 2003; 5: 175-186.

15 Bullard B, Lipski S, Lafontaine ER. Regions important for the adhesin activity of Moraxella catarrhalis Hag. BMC Microbiol 2007; 7: 65.

16 Hoiczyk E, Roggenkamp A, Reichenbecher M, et al. Structure and sequence analysis of Yersinia YadA and Moraxella UspAs reveal a novel class of adhesins. EMBO J 2000; 19: 5989-5999.

17 Singh B, Alvarado-Kristensson M, Johansson M, et al. The respiratory pathogen Moraxella catarrhalis targets collagen for maximal adherence to host tissues. MBio 2016; 7: e00066.

18 Tan TT, Nordström T, Forsgren A, et al. The respiratory pathogen Moraxella catarrhalis adheres to epithelial cells by interacting with fibronectin through ubiquitous surface proteins A1 and A2. J Infect Dis 2005; 192: 1029-1038.

19 Arhin A, Boucher C. The outer membrane protein OprQ and adherence of Pseudomonas aeruginosa to human fibronectin. Microbiology 2010; 156: 1415-1423.

20 Su YC, Mukherjee O, Singh B, et al. Haemophilus influenzae P4 interacts with extracellular matrix proteins promoting adhesion and serum resistance. J Infect Dis 2016; 213: 314-323.

21 Hallström T, Blom AM, Zipfel PF, et al. Nontypeable Haemophilus influenzae protein E binds vitronectin and is important for serum resistance. J Immunol 2009; 183: 2593-2601.

22 Singh B, Al-Jubair T, Mörgelin M, et al. The unique structure of Haemophilus influenzae protein E reveals multiple binding sites for host factors. Infect Immun 2013; 81: 801-814.

23 Attia AS, Ram S, Rice PA, et al. Binding of vitronectin by the Moraxella catarrhalis UspA2 protein interferes with late stages of the complement cascade. Infect Immun 2006; 74: 1597-1611.

24 Singh B, Blom AM, Unal C, et al. Vitronectin binds to the head region of Moraxella catarrhalis ubiquitous surface protein A2 and confers complement-inhibitory activity. Mol Microbiol 2010; 75: 1426-1444. 
25 Paulsson M, Singh B, Al-Jubair T, et al. Identification of outer membrane Porin D as a vitronectin-binding factor in cystic fibrosis clinical isolates of Pseudomonas aeruginosa. J Cyst Fibros 2015; 14: 600-607.

26 Su YC, Jalalvand F, Mörgelin M, et al. Haemophilus influenzae acquires vitronectin via the ubiquitous Protein F to subvert host innate immunity. Mol Microbiol 2013; 87: 1245-1266.

27 Hallström T, Uhde M, Singh B, et al. Pseudomonas aeruginosa uses dihydrolipoamide dehydrogenase (Lpd) to bind to the human terminal pathway regulators vitronectin and clusterin to inhibit terminal pathway complement attack. PLoS One 2015; 10: e0137630.

28 Tan TT, Forsgren A, Riesbeck K. The respiratory pathogen Moraxella catarrhalis binds to laminin via ubiquitous surface proteins A1 and A2. J Infect Dis 2006; 194: 493-497.

29 Su YC, Halang P, Fleury C, et al. Haemophilus protein F orthologs of pathogens infecting the airways: exploiting host laminin at heparin-binding sites for maximal adherence to epithelial cells. J Infect Dis 2017; 216: 1303-1307.

30 Hallström T, Singh B, Resman F, et al. Haemophilus influenzae protein E binds to the extracellular matrix by concurrently interacting with laminin and vitronectin. I Infect Dis 2011; 204: 1065-1074.

31 Jalalvand F, Su YC, Mörgelin M, et al. Haemophilus influenzae protein F mediates binding to laminin and human pulmonary epithelial cells. J Infect Dis 2013; 207: 803-813.

32 Booth AJ, Hadley R, Cornett AM, et al. Acellular normal and fibrotic human lung matrices as a culture system for in vitro investigation. Am J Respir Crit Care Med 2012; 186: 866-876.

33 Shoulders MD, Raines RT. Collagen structure and stability. Annu Rev Biochem 2009; 78: 929-958.

34 Abdillahi SM, Bober M, Nordin S, et al. Collagen VI is upregulated in COPD and serves both as an adhesive target and a bactericidal barrier for Moraxella catarrhalis. J Innate Immun 2015; 7: 506-517.

35 Bresser P, Virkola R, Jonsson-Vihanne M, et al. Interaction of clinical isolates of nonencapsulated Haemophilus influenzae with mammalian extracellular matrix proteins. FEMS Immunol Med Microbiol 2000; 28: 129-132.

36 Spahich NA, St Geme JW 3rd. Structure and function of the Haemophilus influenzae autotransporters. Front Cell Infect Microbiol 2011; 1: 5.

37 Spahich NA, Kenjale R, McCann J, et al. Structural determinants of the interaction between the Haemophilus influenzae Hap autotransporter and fibronectin. Microbiology 2014; 160: 1182-1190.

38 Tan TT, Christensen JJ, Dziegiel MH, et al. Comparison of the serological responses to Moraxella catarrhalis immunoglobulin D-binding outer membrane protein and the ubiquitous surface proteins $\mathrm{A} 1$ and $\mathrm{A} 2$. Infect Immun 2006; 74: 6377-6386.

39 Su YC, Hallström BM, Bernhard S, et al. Impact of sequence diversity in the Moraxella catarrhalis UspA2/ UspA2H head domain on vitronectin binding and antigenic variation. Microbes Infect 2013; 15: 375-387.

40 Tsang KW, Shum DK, Chan S, et al. Pseudomonas aeruginosa adherence to human basement membrane collagen in vitro. Eur Respir J 2003; 21: 932-938.

41 Tsang KW, Rutman A, Tanaka E, et al. Interaction of Pseudomonas aeruginosa with human respiratory mucosa in vitro. Eur Respir J 1994; 7: 1746-1753.

42 Henderson B, Nair S, Pallas J, et al. Fibronectin: a multidomain host adhesin targeted by bacterial fibronectin-binding proteins. FEMS Microbiol Rev 2011; 35: 147-200.

43 Kluftinger JL, Kelly NM, Jost BH, et al. Fibronectin as an enhancer of nonopsonic phagocytosis of Pseudomonas aeruginosa by macrophages. Infect Immun 1989; 57: 2782-2785.

44 Gawronski JD, Wong SM, Giannoukos G, et al. Tracking insertion mutants within libraries by deep sequencing and a genome-wide screen for Haemophilus genes required in the lung. Proc Natl Acad Sci USA 2009; 106 : $16422-16427$.

45 Spaniol V, Troller R, Aebi C. Physiologic cold shock increases adherence of Moraxella catarrhalis to and secretion of interleukin 8 in human upper respiratory tract epithelial cells. I Infect Dis 2009; 200: 1593-1601.

46 Paulsson $\mathrm{M}$, Che $\mathrm{K}$, Ahl J, et al. Bacterial outer membrane vesicles induce vitronectin release into the bronchoalveolar space conferring protection from complement-mediated killing. Frontiers Microbiol 2018; in press.

47 Hallström T, Singh B, Kraiczy P, et al. Conserved patterns of microbial immune escape: pathogenic microbes of diverse origin target the human terminal complement inhibitor vitronectin via a single common motif. PLoS One 2016; 11: e0147709.

48 Ikeda M, Enomoto N, Hashimoto D, et al. Nontypeable Haemophilus influenzae exploits the interaction between protein-E and vitronectin for the adherence and invasion to bronchial epithelial cells. BMC Microbiol 2015; 15: 263.

49 Juschten J, Tuinman PR, Juffermans NP, et al. Nebulized anticoagulants in lung injury in critically ill patients an updated systematic review of preclinical and clinical studies. Ann Transl Med 2017; 5: 444.

50 IPHIVAP investigators of the Australian and New Zealand Intensive Care Society Clinical Trials Group. Is inhaled prophylactic heparin useful for prevention and management of pneumonia in ventilated ICU patients? J Crit Care 2016; 35: 231-239. 